\title{
Evaluation of Disuse Atrophy of Rat Skeletal Muscle Based on Muscle Energy Metabolism Assessed by ${ }^{31}$ P-MRS
}

\author{
Naoyuki Yoshida' ${ }^{1)}$, Takaaki Ikata1), Koichi Sairyo ${ }^{1)}$, Tetsuya Matsuura ${ }^{1)}$, \\ Takahiro Sasa ${ }^{1)}$, Keiko Koga ${ }^{2)}$ and Mari Fukunaga ${ }^{2)}$ \\ 1) Department of Orthopedic Surgery, School of Medicine, The University of Tokushima \\ 2) Tokushima Research Institute, Otsuka Pharmaceutical Co., Ltd.
}

\begin{abstract}
The purpose of this study was to evaluate disuse atrophy of skeletal muscle using a hind-limb suspension model, with special reference to energy metabolism. Twenty-four Sprague-Dawley rats were divided into four groups: control group (C), hind-limb suspended for 3 days (HS-3), for 7 days (HS-7) and for 14 days (HS-14). The gastrocnemius-plantaris-soleus (GPS) muscles in each group were subjected to the following measurements. After a 2-min rest, contraction of the GPS muscles was induced by electrical stimulation of the sciatic nerve at $0.25 \mathrm{~Hz}$ for $10 \mathrm{~min}$, then the frequency was increased to 0.5 and $1.0 \mathrm{~Hz}$ every $10 \mathrm{~min}$. During the stimulation, twitch forces were recorded by a strain gauge, and ${ }^{31} \mathrm{P}-\mathrm{MRS}$ was performed simultaneously. Maximum tension was measured at the muscle contraction induced at $0.25 \mathrm{~Hz}$; the wet weight of the whole and each muscle in the GPS muscles was also measured. From the ${ }^{31} \mathrm{P}-\mathrm{MR}$ spectra during muscle contraction, the oxidative capacity was calculated and compared among the groups. The weights of the whole GPS muscles in C, HS-3, HS-7 and HS-14, were $2.66 \pm 0.09$, $2.39 \pm 0.21,2.34 \pm 0.21$ and $2.18 \pm 0.14$ (g) respectively. Thus, the muscle mass significantly decreased with time $(p<0.05)$. Among the GPS muscles, the decrease in weight of the soleus muscle was especially remarkable; in the HS-14 group its weight decreased to $60 \%$ of that in the $\mathrm{C}$ group. We evaluated maximum tension and oxidative capacity as the muscle function. The maximum tensions in C, HS-3, HS-7 and HS-14 were $519 \pm 43,446 \pm 66,450 \pm$ 23 and $465 \pm 29$ (g), respectively. This was significantly greater in the $\mathrm{C}$ group than in any other groups, however there were no significant differences among the three HS groups. The oxidative capacity during muscle contraction in the $\mathrm{C}$ group was higher than in any HS group and it did not further decrease even if the suspension of the limbs was prolonged beyond 3 days. The present study showed that in disuse atrophy, muscle
\end{abstract}

mass and muscle function did not change simultaneously. Thus, it is necessary to develop countermeasures to prevent muscle atrophy and muscle function deterioration independently. JPhysiol Anthropol 20 (4): 247-252, 2001 http://www.jstage.jst.go.jp/en/

Keywords: disuse atrophy, muscle, energy metabolism, ${ }^{31} \mathrm{P}-\mathrm{MRS}$

\section{Introduction}

Recently, since muscle disuse atrophy after returning to the Earth is crucial for astronauts, the research done up to the present to clarify the pathogenesis of the atrophy has been highlighted by many physiologists, and countermeasures to prevent atrophy, such as doing exercise and stretching during the space mission, have been undertaken (Keitenberg et al., 1998). In the field of rehabilitation as well, disuse atrophy of skeletal muscles has been one of the problems which has yet to be resolved. During joint immobilization in the treatment for fractures or during the bed resting period following surgery, disuse atrophy certainly occurs and it makes subsequent rehabilitation difficult (Berg et al. 1991; Häggmark et al. 1986). To establish optimal countermeasures for a smoothly managed rehabilitation, we need to clarify pathogenesis of disuse muscle atrophy completely.

Although much has been reported about disuse atrophy of skeletal muscle and the pathogenesis of atrophy (Alford et al., 1987; Baldwin et al., 1993; Berg et al., 1991; Desplanches et al., 1990; Fell et al., 1985; Gardetto et al., 1989; Goldspink et al., 1986; Krieger et al., 1980; Levio et al., 1998; Marsh et al., 1992; Oishi et al., 1998; Riley et al., 1990; Steffen and Musacchia, 1984; Thomason and Booth, 1990; Yajid et al., 1998) since the pioneering efforts of 
Morey (1979) and Musacchia et al. (1980), the mechanism has yet to be fully understood. Intramuscular energy metabolism needs to be measured in vivo, and it is important to examine the changes in metabolism in the working muscle, because skeletal muscles are the organs for exercising and working. After phosphorus-31 magnetic resonance spectroscopy $\left({ }^{31} \mathrm{P}-\mathrm{MRS}\right)$ was applied to the skeletal muscle, a number of studies has been conducted and intramuscular energy metabolism has been evaluated under many skeletal muscle conditions (Chance et al., 1981; Iwanaga et al., 1992; Kato et al., 2000; Kemp et al., 1996; Meyer, 1988; McCully et al., 1989; Sairyo et al., 1993).

So far, lots of in vitro studies have focused on the muscle energy metabolism (Baldwin et al., 1993; Desplanches et al., 1990; Fell et al., 1985; Krieger et al., 1980; Levio et al., 1998; Roy et al., 1987) of muscles affected by disuse atrophy, and have indicated that oxidative capacity decreased in the case of disuse. However, the course of the decreasing process has not been investigated as yet.

The purpose of this study was, thus, to confirm the deterioration of the oxidative capacity in the disuse atrophied muscle in vivo using ${ }^{31} \mathrm{P}-\mathrm{MRS}$, and to evaluate changes with time as well as to investigate changes in muscle volume.

\section{Materials and Methods}

\section{Materials}

The 24 male 14-week old Sprague-Dawley rats used in this study were obtained from SLC Japan Co. Ltd. (Shizuoka, Japan). They were housed in individual cages at a temperature of $22 \pm 1^{\circ} \mathrm{C}$ and humidity $50 \pm 10 \%$ in a controlled room on a 12: 12 hours light-dark cycle. The animals had free access to food and water. They were assigned to four groups: six rats to the control group (C), six were subjected to hind-limb suspension for 3 days (HS-3 group), six for 7 days (HS-7 group) and six for 14 days (HS-14 group).

\section{Hind-limb suspension procedure}

The suspension method established in our laboratory (Fukuta et al., 1992; Matsuura et al., in press) was a modification of Morey's tail suspension model (Morey, 1979). After the rat was anesthetized with sodium pentobarbital (50 mg/kg body weight), a harness was glued and attached to the proximal two thirds of the tail. The harness was then connected to a stainless steel wire, and the wire was attached to the top of the cage by a ballbearing wheel swivel, which allowed the rat to rotate in 360-degrees. The suspension height was adjusted to prevent the hindlimb from contacting the floor or sides of the cage while the forelimbs were allowed to support their weight. Despite hindlimb suspension, the rats still had free access to food and water.

\section{Experimental preparation for measurement}

After the rat was anesthetized with sodium pentobarbital (50 mg/kg body weight), the right sciatic nerve was exposed in the gluteal region and a small bipolar electrode was attached to the sciatic nerve. The distal tendon of the GPS muscles were exposed, and cut at the insertion end to the calcaneal bone. The rat was immobilized on a small platform with both the knee and ankle in the full-extended position. A strain gauge (T11000-240, Orientec Co. Ltd., Tokyo, Japan) was attached to the distal tendon. Then, a $20 \times 14 \mathrm{~mm}$ surface coil was placed on the right gastrocnemius-plantaris-soleus (GPS) muscles so that force could be measured at the same time of ${ }^{31} \mathrm{P}-\mathrm{MRS}$ measurement. Then, the platform with the rat was inserted into the magnet.

\section{Experimental protocol}

Following a two-minute rest, contraction of the GPS muscles was induced by electrical stimulation (SEN-3301, Nihon Kohden Co. Ltd., Japan) of the sciatic nerve at 0.25 $\mathrm{Hz}$ for $10 \mathrm{~min}$. Then, the stimulation frequency was increased to 0.5 and $1.0 \mathrm{~Hz}$ every $10 \mathrm{~min}$. Stimulation voltage was $20-30 \mathrm{~V}$ so that supramaximal contraction was produced in all groups at $1.0 \mathrm{~Hz}$. During the stimulation, twitch forces were recorded on a chart recorder, and ${ }^{31} \mathrm{P}-\mathrm{MRS}$ was carried out simultaneously.

${ }^{31} \mathrm{P}-\mathrm{MR}$ spectra were obtained in a $17-\mathrm{cm}$ diameter horizontal bore magnet at 4.7 Tesla (BEM 170/200, Otsuka Electronics USA Inc. USA) at $81.1 \mathrm{MHz}$. The repetition time of pulse was used $2 \mathrm{sec}$. The most suitable pulse duration ( $22 \mu \mathrm{s}$ ) was chosen to obtain the maximum signal to noise ratio. Radio-frequency transmission and signal detection were performed with an oval surface coil $(20 \times 14 \mathrm{~mm})$.

${ }^{31} \mathrm{P}-\mathrm{MR}$ spectra were recorded at rest and during muscle contraction. Based on the spectra recorded during the last 2-min of each stimulation condition, that is, when the consumption of energy reached a steady state, the following measurements were conducted to evaluate energy metabolism of the working muscles. The area of inorganic phosphate $(\mathrm{Pi})$ and phosphocreatine $(\mathrm{PCr})$ were measured to calculate the $\mathrm{PCr} /(\mathrm{Pi}+\mathrm{PCr})$ ratio, which is the indicator of the level of energy state. At rest, the peak of Pi is very small; therefore the peak of Pi is likely to be interfered by noise. Thus, it is not difficult to assulme that the $\mathrm{Pi} / \mathrm{PCr}$ ratio is not reliable at the resting condition. This is the reason why we used $\mathrm{PCr} /(\mathrm{Pi}+\mathrm{PCr})$, not $\mathrm{Pi} / \mathrm{PCr}$ ratio. The areas of ${ }^{31} \mathrm{P}-\mathrm{MR}$ spectra were simulated with a Lorentzian line using a least-squares fit algorithm (FIT R1.2, Otsuka Electronics, Osaka, Japan). The intracellular $\mathrm{pH}$ was also calculated by the chemical shift (d) between Pi and PCr peak as follows (Flaherty et al., 1982): 
Intracellular $\mathrm{pH}=6.90-\log ((\mathrm{d}-6.81) /(3.29-\mathrm{d}))$

The intracellular $\mathrm{pH}$ calculated by this equation seems to have a tendency to be higher than that using another equation (Hope et al. 1984, Kusuoka et al. 1985).

The force time rate (force $\times$ rate) at the steady state obtained from the tension measurement was calculated as the indicator of the momentum of muscle contractions according to the method proposed by Meyer (1988).

Maximum tension of twitch was measured at the muscle contraction induced by $0.25 \mathrm{~Hz}$, and muscle wet weight was also determined in each muscle of the GPS muscles.

\section{Data analysis}

Data were expressed as the mean \pm SD. One way ANOVA, post hoc multiple comparison and analysis of covariance were used for statistical analysis. A p value of less than 0.05 was taken as significant.

\section{Results}

\section{Muscle weight}

The weights of the whole GPS muscles in C, HS-3, HS-7 and HS-14, were $2.66 \pm 0.09,2.39 \pm 0.21,2.34 \pm 0.21$ and $2.18 \pm 0.14$ (g), respectively (Table 1 ). Thus, the muscle mass significantly decreased with time $(p<0.05)$. Among the GPS muscles, the decrease in weight of the soleus muscle was especially remarkable; in the HS-14 group its weight decreased to $60 \%$ of that in the $\mathrm{C}$ group.

\section{Maximum tension}

The maximum tensions in C, HS-3, HS-7 and HS-14 were $519 \pm 43,446 \pm 66,450 \pm 23$ and $465 \pm 29(\mathrm{~g})$, respectively. This was significantly $(p<0.05)$ greater in the $\mathrm{C}$ group than in any other group, however there were no significant differences among the three HS groups.

\section{${ }^{31} P-M R S$ spectrometry}

Representative ${ }^{31} \mathrm{P}-\mathrm{MR}$ spectra are shown in Fig. 1. Intracellular $\mathrm{pH}$ did not decrease below 7.0 during muscle contractions, indicating the muscle exercise was aerobic (Fig. 2). Significant $(p<0.05)$ linear relationships between the $\mathrm{PCr} /(\mathrm{Pi}+\mathrm{PCr})$ ratio and the force $\times$ rate were found in all groups during muscle contraction at the steady state under each stimulation condition (Fig. 3). The slope in C group was the gentlest ( $p<0.05$; analysis of covariance); however there were no significant differences among the three HS groups.

\section{Discussion}

Hind-limb suspension induces hypokinesia and hypodynamia of the hind-limb muscles. Those conditions, mainly hypodynamia, are considered to be important contributing factors in the development of muscle atrophy (Alford et al., 1987; Riley et al., 1990). Especially, slow type fibers were reported to show the greater sensitivity to the HS rather than fast twitch fibers, thereby it is reported that slow-to-fast conversion was induced by the HS (Marsh et al., 1992; Oishi et al., 1998; Riley et al., 1990). Skeletal muscle atrophy, as evaluated by measuring the muscle wet weight in this study, worsened with time. The decrease was greater in the soleus muscle whose fibers are mostly of the slow type. These data indicated that hind-limb suspension induced muscle atrophy, and that disuse affected mainly the muscle constituted by slow type fibers. The present results were in agreement with those of the previous reports, and also indicated that the model in this study was appropriate for investigating disuse muscle atrophy.

As for skeletal muscle function parameters, we measured maximum tension and intramuscular energy metabolism. The maximum twitch and tetanic tension were reported to decrease after HS (Gardetto et al., 1989). This effect was induced by an increased protein degradation and decreased protein synthesis (Goldspink et al., 1986; Loughna et al., 1986; Steffen et al., 1984). Concerning the oxidative capacity, in vitro studies revealed that the capacity decreased by disuse (Fell et al., 1985; Baldwin et al., 1993; Fell et al., 1985; Krieger et al., 1980; Roy et al., 1987). However, the course of the decreasing process with time has not been investigated as yet.

The energy metabolism was evaluated in vivo using ${ }^{31} \mathrm{P}$ MRS in this study. Intracellular $\mathrm{pH}$ during the electrical stimulation at any frequencies never decreased below 7.0

Table 1 Body and muscle wet weight

\begin{tabular}{|c|c|c|c|c|c|}
\hline \multirow[t]{2}{*}{ Group } & \multirow[t]{2}{*}{ Body weight (g) } & \multicolumn{4}{|c|}{ Muscle wet weight (mg) } \\
\hline & & Total & Soleus & Plantaris & Gastrocnemius \\
\hline Control & $410 \pm 10$ & $2.66 \pm 0.09$ & $0.17 \pm 0.01$ & $0.42 \pm 0.04$ & $2.07 \pm 0.07$ \\
\hline HS-3 & $381 \pm 12^{*}$ & $2.39 \pm 0.21^{*}$ & $0.15 \pm 0.02^{*}$ & $0.40 \pm 0.04$ & $1.84 \pm 0.16^{*}$ \\
\hline HS-7 & $382 \pm 3^{*}$ & $2.34 \pm 0.21^{\star}$ & $0.13 \pm 0.01^{\star} \#$ & $0.39 \pm 0.04^{*}$ & $1.82 \pm 0.17 \#^{\wedge}$ \\
\hline HS-14 & $396 \pm 24$ & $2.18 \pm 0.14^{\star} \#^{\wedge}$ & $0.10 \pm 0.01^{\star} \#^{\wedge}$ & $0.38 \pm 0.03^{*}$ & $1.70 \pm 0.11^{\star} \#^{\wedge}$ \\
\hline
\end{tabular}

Values are means \pm S.D. ${ }^{*} \mathrm{p}<0.05$, compared with Control; $\# \mathrm{p}<0.05$, compared with HS-3; and ${ }^{\wedge} \mathrm{p}<0.05$, compared with HS-7. 


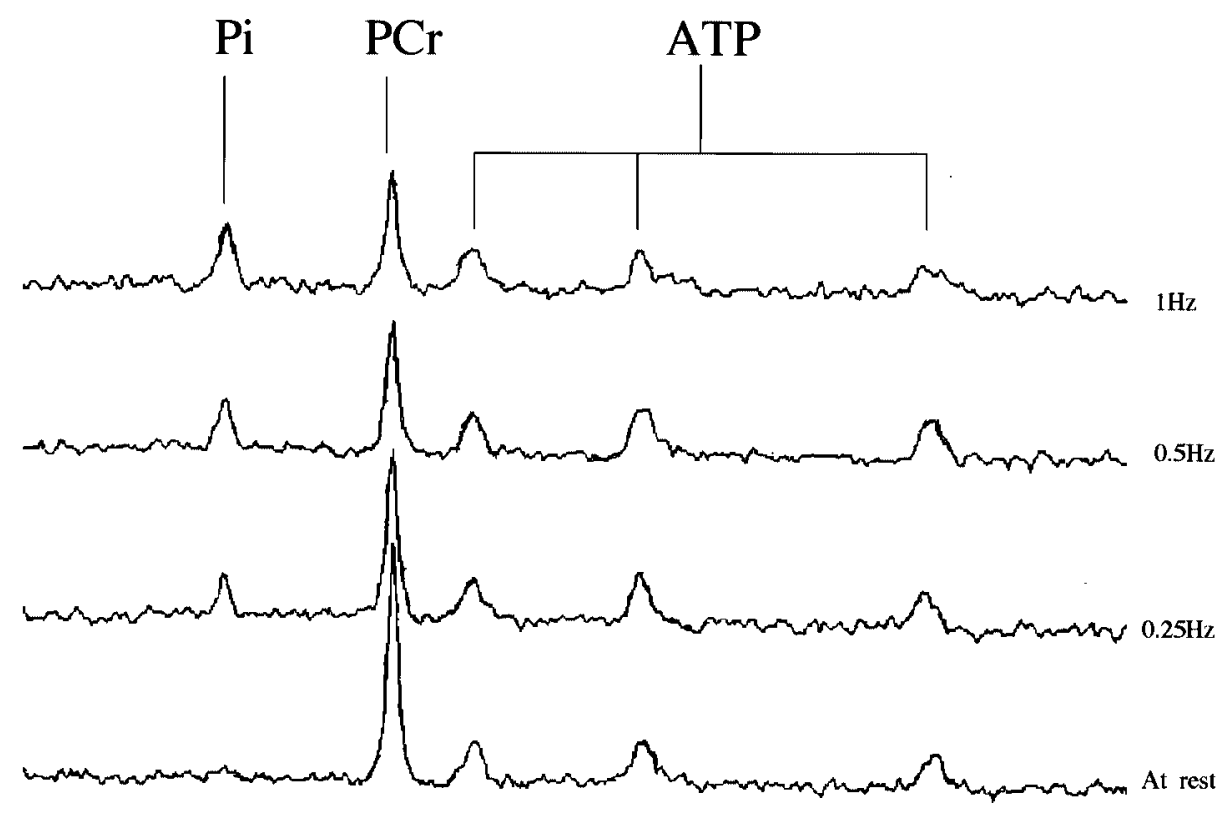

Fig. 1 A typical set of ${ }^{31} \mathrm{P}-\mathrm{MRS}$ spectra at rest and at steady state of each stimulation. Pi, inorganic phosphate; PCr, phosphocreatine; and ATP, adenosine triphosphate.

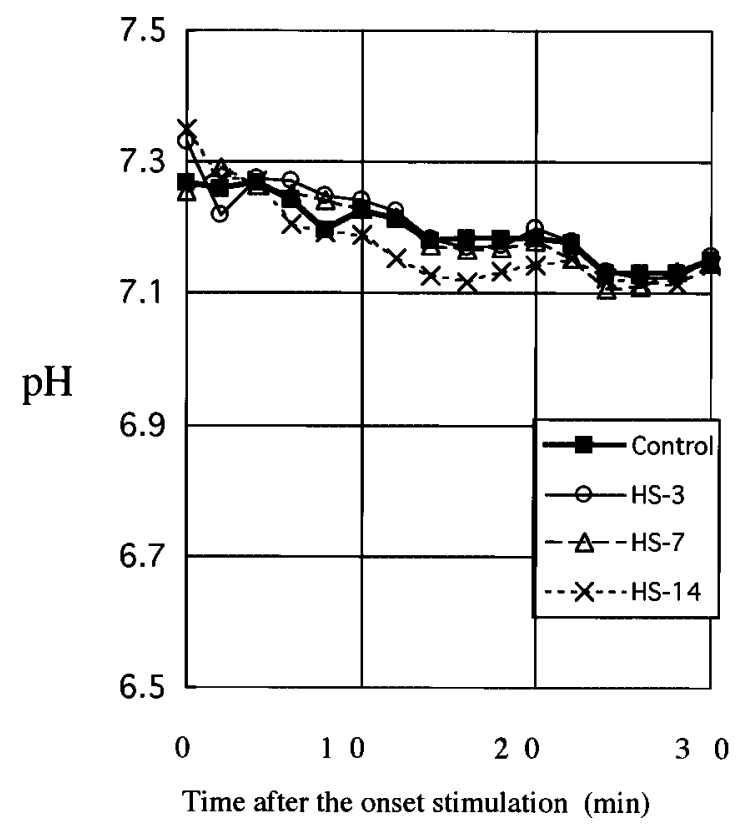

Fig. 2 Time course of change in the $\mathrm{pH}$ at rest and during stimulation. Intracellular $\mathrm{pH}$ did not decrease below 7.0 during the stimulation.

in all groups although it was lower than that at rest, indicated the exercise evoked by the stimulation was aerobic. In such working condition, most energy is supplied from the aerobic metabolism such as oxidative phosphorylation; therefore, the relationship between

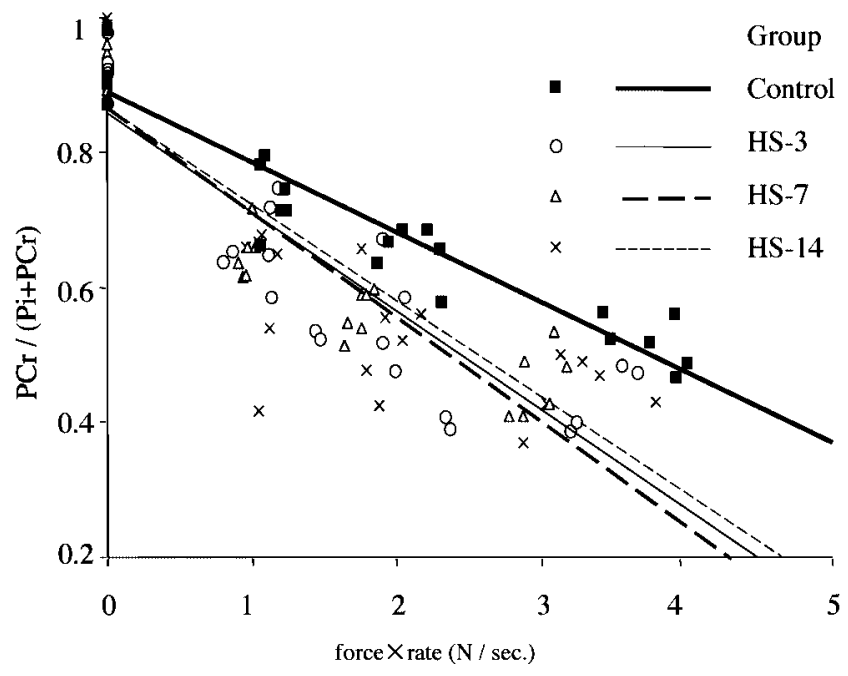

Fig. 3 Regression lines between $\mathrm{PCr} /(\mathrm{Pi}+\mathrm{PCr})$ and force $\times$ rate. The slope in $\mathrm{C}$ group was the gentlest $(\mathrm{p}<0.05)$, and no difference was found among the three HS groups.

workload and $\mathrm{Pi} / \mathrm{PCr}$ ratio reflects oxidative capacity (Chance et al., 1981; McCully et al., 1989). Experimental studies also demonstrated that during muscle twitch stimulation, where the intracellular $\mathrm{pH}$ does not decrease, the relationship indicates muscle oxidative capacity (Kemp et al., 1996; Meyer, 1988). Sasa et al. (2001) evaluated the effects of ovariectomy on skeletal muscle oxidative capacity using this method. We also used this method evaluating oxidative capacity in this 
study, and found that the capacity in the C group was higher than in any HS group and it did not further decrease even if the suspension of the limbs was prolonged beyond 3 days. The maximum twitch tension showed the same time course change as that in the oxidative capacity. Thus, the muscular function decreased with HS as the previous studies indicated; however, the function did not decrease any further after three days of hindlimb suspension. Thus, we evaluated the muscle oxidative capacity using the method proposed by Meyer (1988). We also calculated the force time rate to evaluate momentum of muscle contractions according to his method. The force time rate was not normalized by the muscle cross sectional area in this study; therefore, evaluation of the muscle oxidative capacity might not be strictly qualitative. In order to know the exact qualitative oxidative capacity, the force time rate should be normalized by the cross-sectional area.

Muscle wet weight has been reported to change with time in an exponential manner. The value reached a plateau at around 30 days (Thomason and Booth, 1990). The time course change in muscle oxidative capacity has yet to be fully examined. It might show an exponential pattern similar to the one of the change in muscle volume. As for the chronic adaptations of muscle oxidative capacity to muscle immobilization, Yajid et al. (1998) investigated the change at 4 weeks after hindlimb suspension, and demonstrated 59\% decrease in the capacity of the gastrocnemius muscle. Desplanches et al. (1990) also confirmed effects of 5-wks suspension on the oxidative capacity, and reported $26 \%$ decrease of the subsarcolemmal mitochondrial volume density. On the other hand, Levio et al. (1998) demonstrated that mitochondrial abnormalities might occur within the first few hours of immobilization as shown by a decrease of NADH-TR activity at subsarcolemmal locations being decreased by 10 hours and disappeared by 24 hours of immobilization. Krieger et al. (1980) also investigated the short-term effect of immobilization on muscle oxidative capacity, and found that even for two days of immobilization a significant 37\% decrease in state III respiration of subsarcolemmal mitochondria occurred. Thus, muscle oxidative capacity may be greatly affected at the early stages of immobilization. From the results obtained in the present study, the muscle oxidative capacity decreases with time showing an exponential pattern; i.e. it significantly decreased on day-3, then it reached a plateau. Further studies are required to elucidate the discrepancy between changes in muscle volume and oxidative capacity with time.

In the hind-limb suspension model, hypokinesia of the hind-limb muscles, which was measured as total electromyogram (EMG) activity, improved a few days after the suspension (Alford et al., 1987). The study showed that the EMG activity of the soleus and gastrocnemius muscle decreased only at the beginning to 3 days, indicating that under unweighting conditions as in the case of HS, hypodynamia continues eternally, whereas hypokinesia does not appear except for the initial few days after the start of suspension. The recovery from hypokinesia after a couple of days may greatly contribute to prevent a further decrease of the muscle function such as maximum tension and oxidative capacity, as observed in this study. These data indicated that absence of hypokinesia may minimize functional deterioration during the disuse period, and that active muscle isometric and/or isotonic exercise could be a recommendable manoeuvre for rehabilitation during joint immobilization and bed resting period. Muscle atrophy developed after three days suspension in this study. Therefore, another countermeasure such as stretching and electrical stimulation should be considered to prevent a reduction of muscle volume.

The present study showed that in disuse atrophy, muscle mass and muscle function did not change simultaneously. Thus, it is necessary to develop countermeasures to prevent muscle atrophy and muscle function deterioration independently.

\section{References}

Alford EK, Roy RR, Hodgson JA, Edgerton VR (1987) Electromyography of rat soleus, medial gastrocnemius, and tibialis anterior during hindlimb suspension. Exp Neurol 96: 635-649

Baldwin KM, Herrick RE, McCue SA (1993) Substrate oxidation capacity in rodent skeletal muscle: Effects of exposure to zero gravity. J Appl Physiol 75: 2466-2470

Berg HE, Dudley GA, Häggmark T, Ohlsen H, Tesch PA (1991) Effect of lower limb unloading on skeletal muscle mass and function in human. J Appl Physiol 70: $1882-1885$

Chance B, Leigh Jr. JS, Kent JK, McCully KK, Nioka S, Clark BJ, Maris JM, Graham T (1981) Multiple controls of oxidative metabolism of living tissues as studied by ${ }^{31}$ P-MRS. Proc Natl Acad Sci USA 83: 6714-6718

Desplanches D, Kayar SR, Sampore B, Flandrois R, Hoppeler H (1990) Rat soleus ultrastructure after hindlimb suspension. J Appl Physiol 69: 504-508

Fell RD, Steffen JM, Musacchia XJ (1985) Effect of hypokinesia-hypodynamia on rat muscle oxidative capacity and glucose uptake. Am J Physiol 249: R308R312

Flaherty JT, Weisfeldt ML, Bulkley BH, Gardner TJ, Gott VL, Jacobus WE (1982) Mechanisms of ischemic myocardial cell damage assessed by phosphorus-31 nuclear magnetic resonance. Circulation 65: 561-571

Fukuta S, Ikata T, Miura I (1992) Effect of disuse on muscle energy metabolism studied by in vivo 31 Phosphorus magnetic resonance spectroscopy. 
Zoological Science 9: 947-954

Gardetto PR, Schluter JM, Fitts RH (1989) Contractile function of single muscle fibers after hindlimb suspension. J Appl Physiol 66: 2739-2749

Goldspink DF, Morton AJ, Loughna P, Goldspink G (1986) The effect of hypodynamia on protein turnover and the growth of four skeletal muscles of the rat. Pfluegers Arch 407: 333-340

Häggmark T, Eriksson E, Jansson E (1986) Muscle fiber type changes in human skeletal muscle at injuries and immobilization. Orthopedics 9: 181-185

Hope PL, Costello AM, Cady EB, Deply DT, Tofts PS, Chu A, Hamilton PA, Reynolds EO, Wilkie DR (1984) Cerebral energy metabolism studied with phosphorus NMR spectroscopy in normal and birth-asphyxiated infants. Lancet 18: 366-370

Iwanaga K, Sakurai M, Minami T, Kato Y, Sairyo K (1992) Thresholds for decrease in intracellular $\mathrm{pH}$ and increase in blood lactate during progressive exercise: ${ }^{31} \mathrm{P}-\mathrm{MRS}$ study. Ann Physiol Anthrop 11: 641-648

Kato Y, Ikata T, Takai H, Takata S, Sairyo K, Iwanaga K (2000) Effects of specific warm-up at various intensities on energy metabolism during subsequent exercise. J Sports Med Physical Fitness 40: 126-130

Kemp GJ, Sanderson AL, Thompson GH, Radda GK (1996) Regulation of oxidative and glycogenolytic ATP synthesis in exercising rat skeletal muscle studied by ${ }^{31} \mathrm{P}$ magnetic resonance spectroscopy. NMR in Biomed 9: 261-270

Kreitenberg A, Baldwin KM, Bagian JP, Cotton S, Witmer J, Caiozzo VJ (1998) The "Space Cycle" self powered human centrifuge: A proposed countermeasure for prolonged human space flight. Aviat Space Environ Med 69: 66-72

Krieger DA, Tate CA, McMillin-Wood J, Booth FW (1980) Population of rat skeletal muscle mitochondria after exercise and immobilization. J Appl Physiol 48: 23-28

Kusuoka H, Inoue M, Tsuneoka Y, Watari H, Hori M, Abe H (1985) Augmented energy consumption during early systole as a mechanism of cyclical changes in highenergy phosphates in myocardium assessed by phosphorus nuclear magnetic resonance. Jpn Circ J 49: 1099-1107

Levio I, Kauhanen S, Michelsson JE (1998) Abnormal mitochondria and sarcoplasmic changes in rabbit skeletal muscle induced by immobilization. APMIS 106: 1113-1123

Loughna P, Goldspink G, Goldspink DF (1986) Effect of inactivity and passive stretch on protein turnover in phasic and postural rat muscles. J Appl Physiol 61: 173179

Marsh DR, Campbell CB, Spriet, LL (1992) Effect of hindlimb unweighting on anaerobic metabolism in rat skeletal muscle. J Appl Physiol 72: 1304-1310
Matsuura T, Ikata T, Takata S, Kashiwaguchi S, Niwa M, Sogabe T, Koga K (2001) Effect of weight-bearing on recovery from nerve injury in skeletal muscle. J Appl Physiol (in press)

McCully KK, Barry PB, Matthew T, Michael RF, Chance B (1989) Wrist flexor muscles of elite rowers measured with magnetic resonance spectroscopy. J Appl Physiol 67: 926-932

Meyer RA (1988) A linear model of muscle respiration explains monoexponential phosphocreatine changes. Am J Physiol 254: C548-C553

Morey ER (1979) Spaceflight and bone turnover: Correlation of a new rat model of weightlessness. Bioscience 29: 168-172

Musacchia XJ, Deavers DR, Meininger GA, Davis TP (1980) A model for hypokinesia: Effects on muscle atrophy in the rat. J Appl Physiol 48: 479-486

Oishi Y, Ishihara A, Yamamoto H, Miyamoto E (1998) Hindlimb suspension induces the expression of multiple myosin heavy chain isoforms in single fibers of the rat soleus muscle. Acta Physiol Scand 162: 127-134

Riley DA, Slocum GR, Bain JLW, Sedlak FR, Sowa TE, Mellender JW (1990) Rat hindlimb unloading: soleus histochemistry, ultrastructure, and electromyography. J Appl Physiol 69: 58-66

Roy RR, Bello MA, Bouissou P, Edgerton VR (1987) Size and metabolic properties of fibers in rat fast-twitch muscles after hindlimb suspension. J Appl Physiol 62: 2348-2357

Sairyo K, Ikata T, Takai H, Iwanaga K (1993) Effect of active recovery on intracellular $\mathrm{pH}$ following muscle contraction: A ${ }^{31} \mathrm{P}-\mathrm{MRS}$ study. Ann Physiol Anthrop 12: 173-179

Sasa T, Sairyo K, Yoshida N, Ishikawa M, Fukunaga M (2001) Effects of ovariectomy on intramuscular energy metabolism in young rats. J Physiol Anthrop 20: 71-75

Steffen JM, Musacchia (1984) Effect of hypokinesia and hypodynamia on protein, RNA, and DNA in rat hindlimb muscles. Am J Physiol 247: R728-R732

Thomason DB, Booth FW (1990) Atrophy of the soleus muscle by hindlimb unweighting. J Appl Physiol 68: 112

Yajid F, Mercier JG, Mercier BM, Dubouchaud H, Prefaut C (1998) Effect of four weeks of hindlimb suspension on skeletal muscle mitochondria respiration in rats. J Appl Physiol 84: 479-485

Received: January 5, 2001

Accepted: May 17, 2001

Correspondence to: Koichi Sairyo, Department of Orthopedic Surgery, School of Medicine, The University of Tokushima, 3-18-15 Kuramoto, Tokushima 770-8503, Japan

E-mail: sairyo@clin.med.tokushima-u.ac.jp 